\title{
IN WHICH METRIC SPACES ARE PARALLEL BODIES OF CLOSED SETS CLOSED?
}

\author{
by GERALD BEER
}

(Received 1st March 1982)

\section{Preliminaries}

Let $X$ be a metric space with metric $d$ and for each $x$ in $X$ let $B_{\lambda}[x]$ denote the closed ball of radius $\lambda$ about $x$. Following Valentine [15] if $K \subset X$ and $\lambda$ is positive, then we call the set $B_{\lambda}[K]=\bigcup_{x \in K} B_{\lambda}[x]$ the $\lambda$-parallel body of $K$. The following fact is obvious.

Lemma 1. Let $X$ be a metric space in which each closed and bounded set is compact. Then for each closed (resp. compact) subset $K$ of $X$ and each $\lambda>0$ the parallel body $B_{\lambda}[K]$ is a closed (resp. compact) set.

It is clear that the metric spaces in which each parallel body of each compact set is again compact are precisely those in which each closed and bounded set is compact. Such spaces have been characterised by Hindman [12]. It is the main purpose of this article to characterise those metric spaces in which each parallel body of each closed set is closed.

One characterisation will involve the upper semicontinuous extended real valued functions on $X$. Recall that $f: X \rightarrow[-\infty, \infty]$ is u.s.c. if and only if for each $\alpha \in R$ $f^{-1}([-\infty, \alpha))$ is an open set. It is well known that $f$ is u.s.c. if and only if its hypograph

$$
\text { hypo } f=\{(x, \alpha): \alpha \in R \text { and } \alpha \leqq f(x)\}
$$

is a closed subset of $X \times R$. Let us metrise $X \times R$ in a manner compatible with the product uniformity. For definiteness and computational simplicity we shall use the metric $\rho$ defined by

$$
\rho\left[\left(x_{1}, \alpha_{1}\right),\left(x_{2}, \alpha_{2}\right)\right]=\max \left\{d\left(x_{1}, x_{2}\right),\left|\alpha_{1}-\alpha_{2}\right|\right\} .
$$

Relative to this metric we can take parallel bodies of subsets of $X \times R$; in particular we can take parallel bodies of hypographs of real valued upper semicontinuous functions. Given such a function $f$ and $\lambda>0$ we call the "upper ridge" of $B_{\lambda}$ [hypo $\left.f\right]$ the upper parallel function $f_{\lambda}^{+}$of $f$. Thus, at each $x$ in $X$ the definition of $\rho$ implies

$$
\begin{aligned}
f_{\lambda}^{+}(x) & =\sup \left\{\alpha:(x, \alpha) \in B_{\lambda}[\text { hypo } f]\right\} \\
& =\sup \left\{f(y)+\lambda: y \in B_{\lambda}[x]\right\}
\end{aligned}
$$


Note that if $B_{\lambda}$ [hypo $f$ ] is a closed set, then $f_{\lambda}^{+}$is u.s.c. (in the extended sense). In [3] upper parallel functions are used to study two hyperspace topologies for the upper semicontinuous real valued functions on a compact metric space. In that paper it is shown that if $X$ is compact, then whenever $f: X \rightarrow R$ is u.s.c. each upper parallel function $f_{i}^{+}$of $f$ is both u.s.c. and bounded (whether $f$ is bounded or not). Also, if $X$ is compact and $f$ is continuous, then $\left\{f_{\lambda}^{+}\right\}$converges uniformly to $f$ as $\lambda \rightarrow 0$; uniform convergence may fail if $f$ is merely u.s.c. or if in $X$ we only know that closed and bounded sets are compact. It is clear that for each u.s.c. function $f\left\{f_{\lambda}^{+}\right\} \rightarrow f$ pointwise as $\lambda \rightarrow 0$ without restriction on $X$.

A second characterisation will be given in terms of the behaviour of certain multifunctions on $X$. Following the notation of Nadler [14] let $C L(Y)$ denote the space of nonempty closed subsets of a metric space $Y$. The following definitions of upper and lower semicontinuity for multifunctions are as popular as any.

Definition. Let $X$ and $Y$ be metric spaces. A multifunction $\Gamma: X \rightarrow C L(Y)$ is called u.s.c. (resp. l.s.c.) at $x$ in $X$ if for each open set $V$ satisfying $\Gamma(x) \subset V$ (resp. $\Gamma(x) \cap V \neq \varnothing$ ) there exists a neighbourhood $U$ of $x$ such that for each $y$ in $U \Gamma(y) \subset V$ (resp. $\Gamma(y) \cap V \neq \varnothing)$.

Just as useful for our purposes are the following definitions that appear in Berge [4].

Definition. Let $X$ and $Y$ be metric spaces. A multifunction $\Gamma: X \rightarrow C L(Y)$ is called u.s.c. (resp. l.s.c.) at $x$ in $X$ if for each $\varepsilon>0$ there exists a neighborhood $U$ of $x$ such that for each $y$ in $U \Gamma(y) \subset B_{\varepsilon}[\Gamma(x)]$ (resp. $\left.\Gamma(x) \subset B_{\varepsilon}[\Gamma(y)]\right)$.

In the literature Berge's definitions are often referred to as Hausdorff semicontinuity (see, e.g., [2] or [7]). For compact valued multifunctions the above two definitions of semicontinuity are equivalent. In general Berge upper semicontinuity is implied by the usual notion of upper semicontinuity whereas Berge lower semicontinuity implies the first notion of lower semicontinuity. In the context of complete metric spaces Dolecki and Rolewicz [7] have characterised those multifunctions that are u.s.c. in the usual sense among those u.s.c. in the sense of Berge. Notice that a compact valued multifunction that is both u.s.c. and l.s.c. is continuous with respect to the Hausdorff metric on $C L(Y)$. When we speak of a continuous compact valued multifunction, the Hausdorff metric topology on $C L(Y)$ shall be understood. We refer the interested reader to Borges [5] for other definitions of semicontinuity.

Lemma 2. Let $X$ be a metric space in which each closed and bounded set is compact. For each $\lambda>0$ the multifunction $\Gamma_{\lambda}: X \rightarrow C L(X)$ defined by $\Gamma_{\lambda}(x)=\{y: d(x, y) \leqq \lambda\}$ is upper semicontinuous.

Proof. First note that $\Gamma_{\lambda}$ is compact valued. Now fix $x$ in $X$. If $\Gamma_{\lambda}$ failed to be u.s.c. at $x$ we could find $\varepsilon>0$ and a sequence $\left\{x_{n}\right\}$ convergent to $x$ such that for each $n$ $\Gamma_{\lambda}\left(x_{n}\right) \not B_{\varepsilon}\left[\Gamma_{\lambda}(x)\right]$. For each $n$ choose $y_{n}$ in $\Gamma_{\lambda}\left(x_{n}\right)-B_{\varepsilon}\left[\Gamma_{\lambda}(x)\right]$. Siince $\bigcup_{n=1}^{\infty} \Gamma_{\lambda}\left(x_{n}\right)$ is a bounded set, by passing to a subsequence we can assume that $\left\{y_{n}\right\}$ converges to some point $y$, and we have $d(y, x) \leqq \lambda$, i.e., $y \in \Gamma_{\lambda}(x)$. Now choose $n$ so large that $d\left(y_{n}, y\right)<\varepsilon$. Clearly, $y_{n}$ must then lie in $B_{\varepsilon}\left[\Gamma_{\lambda}(x)\right]$, a contradiction. 
In the sequel we shall call the multifunction $\Gamma_{\lambda}$ defined in the statement of Lemma 2 a ball multifunction. Ball multifunctions in a normed linear space are u.s.c. if and only if the space is finite dimensional [11].

Example 1. Ball multifunctions for locally compact, sigma compact, complete, connected, locally connected spaces need not be u.s.c.. To see this, let $l_{2}$ be the Hilbert space of square summable real sequences and let $\left\{e_{j}: j \in Z^{+}\right\}$denote the standard orthonormal basis for $l_{2}$. For each $j \in Z^{+}$let $a_{j}=(1+1 / j) e_{j}$ and let $b_{j}=(1 / j) e_{j}$. Let $L$ denote the infinite polygonal path joining 0 to $a_{1}$, then $a_{1}$ to $a_{2}$, then $a_{2}$ to $a_{3}$, etc., and let $W$ be the following starshaped set:

$$
W=\bigcup_{j=1}^{\infty}\left\{\lambda b_{j}: 0 \leqq \lambda \leqq 1\right\}
$$

Finally set $X=L \cup W$. We leave it to the reader to verify that $X$ has the asserted properties. The multifunction $\Gamma_{1}$ fails to be u.s.c. at the origin (in either sense) because for each $j a_{j} \in \Gamma_{1}\left(b_{j}\right)$ whereas $B_{1 / 2}\left[\Gamma_{1}(0)\right] \cap\left\{\alpha_{j}: j \geqq 2\right\}=\varnothing$.

\section{The main results}

Our next lemma is at the heart of the characterisation theorem.

Lemma 3. Let $X$ be a metric space. Let $x$ in $X$ and $\lambda>0$ be arbitrary. If the ball multifunction $\Gamma_{\lambda}$ is u.s.c. at $x$, then each upper parallel function $f_{\lambda}^{+}$of each real valued u.s.c. function $f$ on $X$ is u.s.c. at $x$. Conversely, if each upper parallel function $f_{\lambda}^{+}$of each real valued continuous function $f$ on $X$ is u.s.c. at $x$, then $\Gamma_{\lambda}$ is u.s.c. at $x$.

Proof. Suppose $\Gamma_{\lambda}$ is u.s.c. at $x$. Let $f: X \rightarrow R$ be u.s.c.. For each $y \in \Gamma_{y}(x)$ choose $\delta_{y}>0$ such that if $d(y, z) \leqq \delta_{y}$ then $f(z)<f(y)+\varepsilon$. Now let $V=\bigcup\left\{B_{\delta_{y}}[y]: y \in \Gamma_{\lambda}(x)\right\}$. Since $\Gamma_{\lambda}$ is u.s.c. at $x$ there exists a neighbourhood $U$ of $x$ such that for each $w$ in $U \Gamma_{\lambda}(w) \subset V$. By the construction of $V$ for each $w$ in $U$ we have

$$
\sup \left[f(z): z \in \Gamma_{\lambda}(w)\right\} \leqq \sup \{f(z): z \in V\} \leqq \sup \left\{f(z): z \in \Gamma_{\lambda}(x)\right\}+\varepsilon
$$

By the definition of $f_{\lambda}^{+}$we have $f_{\lambda}^{+}(x)+\varepsilon \geqq f_{\lambda}^{+}(w)$ and $f_{\lambda}^{+}$is u.s.c. at $x$ (although not necessarily finite valued).

Conversely suppose $\Gamma_{\lambda}$ is not u.s.c. at $x$. We can find an open set $V$ containing $\Gamma_{\lambda}(x)$ and a sequence $\left\{x_{n}\right\}$ convergent to $x$ such that for each $n \Gamma_{\lambda}\left(x_{n}\right) \not V$. For each $n$ choose $y_{n} \in \Gamma_{\lambda}\left(x_{n}\right)-V$. Clearly, $\left\{y_{n}\right\}$ can have no convergent subsequence, for the limit would be in $\Gamma_{\lambda}(x)$ so that $\left\{y_{n}\right\}$ would be in $V$ frequently. Without loss of generality we can assume that the terms of the sequence are distinct. For each $n \in Z^{+}$choose $\delta_{n}>0$ such that

(i) $0<\delta_{n}<\min \left\{\frac{1}{n}, d\left(x, y_{n}\right)-\lambda\right\}$

(ii) for each $j \neq n d\left(y_{n}, y_{j}\right)>2 \delta_{n}$. 
It follows that for each $j$ and $k B_{\delta_{j}}\left[y_{j}\right] \cap B_{\delta_{k}}\left[y_{k}\right]=\varnothing$. Set

$$
F=\bigcup_{j=1}^{\infty} B_{\delta_{j}}\left[y_{j}\right]
$$

We claim that $F$ is a closed set. To see this let $\left\{z_{k}\right\}$ be a sequence in $F$ convergent to some point $z$. Clearly, $\left\{z_{k}\right\}$ cannot be in each of two of the balls that comprise $F$ frequently; so, either $\left\{z_{k}\right\}$ is in infinitely many balls or $\left\{z_{k}\right\}$ is in one ball, say $B_{\delta_{n}}\left[y_{n}\right]$, eventually. In the first case since $\left\{\delta_{k}\right\} \rightarrow 0$, a subsequence of $\left\{y_{k}\right\}$ must also converge to $z$, an impossibility. Hence, the second alternative must occur, whence $z \in F$ because $B_{\delta_{n}}\left[y_{n}\right]$ is a closed set. Thus, $F$ is closed. Now define $f: X \rightarrow R$ by the formula

$$
f(z)=\left\{\begin{array}{cl}
1-\frac{1}{\delta_{n}} d\left(z, y_{n}\right) & \text { if for some } n \quad z \in B_{\delta_{n}}\left[y_{n}\right] \\
0 & \text { if } \quad x \notin F
\end{array}\right.
$$

Clearly, $f$ is continuous on $F$ and $c l\left(F^{c}\right)$; so, by a standard criterion [13; p. 33] $f$ is globally continuous. For each $n f_{\lambda}^{+}\left(x_{n}\right)=1+\lambda$ because $f\left(y_{n}\right)=1$. However, $f_{\lambda}^{+}(x)=\lambda$ because the distance of $x$ from each point of $F$ exceeds $\lambda$. Thus, $f_{\lambda}^{+}$is not u.s.c. at $x$.

Theorem 1. Let $X$ be a metric space. The following are equivalent.

(i) Each parallel body of each closed subset of $X$ is closed.

(ii) Each upper parallel function of each real valued u.s.c. function on $X$ is an extended real valued u.s.c. function.

(iii) Each upper parallel function of each real valued continuous function on $X$ is an extended real valued u.s.c. function.

(iv) Each ball multifunction on $X$ is u.s.c..

Proof. The equivalence of (ii), (iii), and (iv) follows from Lemma 3. It is also easy to see that statements (i) and (iv) are equivalent: by the definition of upper semicontinuity $\Gamma_{\lambda}: X \rightarrow C L(X)$ is u.s.c. if and only if for each closed subset $A$ of $X$ the set $\left\{x: \Gamma_{\lambda}(x) \cap A \neq \varnothing\right\}$ is a closed subset of $X$. However, $\left\{x: \Gamma_{\lambda}(x) \cap A \neq \varnothing\right]$ is precisely the $\lambda$-parallel body of $A$.

Are each of the conditions presented in the statement of Theorem 1 equivalent to the following condition: for each $\lambda>0$ and each u.s.c. function $f: X \rightarrow R$ the set $B_{\lambda}$ [hypo $f$ ] is a closed subset of $X \times R$ ? The answer is negative.

Example 2. As in Example 1 let $\left\{e_{j}: j \in Z^{+}\right\}$denote the standard orthonormal basis for $l_{2}$. If we regard this discrete set as a metric subspace $X$ of $l_{2}$, then each parallel body of each closed subset of $X$ is closed. In fact, if $A \subset X$, then $B_{\lambda}[A]$ is either $A$ or $X$. Now define $f: X \rightarrow R$ by

$$
f(x)=\left\{\begin{array}{lll}
-1 / j & \text { if } & x=e_{j} \\
-10 & \text { if } & x=e_{1}
\end{array} \text { for some } j>1\right.
$$


Although $f$ is continuous $B_{\sqrt{2}}[$ hypo $f]$ is not closed, for it fails to contain the limit point $\left(e_{1}, \sqrt{2}\right)$.

It should be noticed that the metric space of Example 2 is one in which not all closed and bounded sets are compact, but is one in which parallel bodies of closed sets are closed.

Theorem 2. Let $X$ be a metric space. The following are equivalent.

(i) Each closed and bounded subset of $X$ is compact.

(ii) For each real valued u.s.c. function $f$ on $X$ and each $\lambda>0$, the set $\beta_{\lambda}[$ hypo $f]$ is $a$ closed subset of $X \times R$.

(iii) Each upper parallel function of each real valued u.s.c. function on $X$ is again a real valued u.s.c. function on $X$.

(iv) Each upper parallel function of each real valued continuous function on $X$ is real valued.

Proof. (i) $\rightarrow$ (ii) Closed and bounded subsets of $X \times R$ must be compact, and the implication follows from Lemma 1.

(ii) $\rightarrow$ (i) If for some $x$ and $\lambda>0$ the ball $B_{\lambda}[x]$ is noncompact, choose a sequence $\left\{y_{n}\right\}$ in the ball with no convergent subsequence, and define $f: X \rightarrow R$ by $f\left(y_{n}\right)=1-1 / n$. and zero otherwise. As in Example 2, $B_{\lambda}[$ hypo $f]$ is nonclosed.

(i) $\rightarrow$ (iii) Let $f: X \rightarrow R$ be u.s.c. By Theorem 1 and Lemma 1 for each $\lambda>0 f_{\lambda}^{+}$is u.s.c. For each $x, \sup \left\{f(y)+\lambda: y \in B_{\lambda}[x]\right\}$ is achieved because $B_{\lambda}[x]$ is compact and $f$ is u.s.c. Thus, $-\infty<f(x)<f_{\lambda}^{+}(x)<\infty$.

(iii) $\rightarrow$ (iv) Obvious.

(iv) $\rightarrow$ (i) It suffices to show that each closed ball in $X$ is compact. Suppose for some $x \in X$ and $\lambda>0$ the ball $B_{\lambda}[x]$ is noncompact. Then we can find a sequence $\left\{y_{n}\right\}$ in the ball with no convergent subsequence. Without loss of generality we can assume that all the terms are distinct. As usual set $E=\left\{y_{n}: n \in Z^{+}\right\}$and define $g: E \rightarrow R$ by $g\left(y_{n}\right)=n$. Since $E$ is closed, the Tietze extension theorem yields an $f$ in $C(X)$ such that $f \mid E=g$. Clearly, $f_{\lambda}^{+}(x)=\infty$.

Lemma 4. Let $X$ be a metric space in which each closed and bounded set is compact, and let $\lambda$ be positive. If $x \in X$ then $\Gamma_{\lambda}$ is discontinuous at $x$ if and only if there exists $f \in C(X)$ such that $f_{\lambda}^{+}$is discontinuous at $x$.

Proof. Suppose that the multifunction $\Gamma_{\lambda}$ is continuous at $x$ but for some $f$ in $C(X)$ the upper parallel function $f_{\lambda}^{+}$is discontinuous at $x$. By Theorem $2 f_{\lambda}^{+}$is an u.s.c. real valued function; so, there exists a sequence $\left\{x_{n}\right\}$ in $X$ convergent to $x$ and an extended real number $\theta<f_{\lambda}^{+}(x)$ such that $\lim _{n \rightarrow \infty} f_{\lambda}^{+}\left(x_{n}\right)=\theta$. By the definition of $f_{\lambda}^{+}$and the compactness of closed $\lambda$-balls in $X$ there exists $y \in \Gamma_{\lambda}(x)$ for which $f_{\lambda}^{+}(x)=f(y)+\lambda$. Choose $\varepsilon>0$ satisfying $\varepsilon<f_{\lambda}^{+}(x)-\theta$ and a positive integer $N$ so large that for each $n \geqq N$

$$
f_{\lambda}^{+}\left(x_{n}\right)<f_{\lambda}^{+}(x)-\frac{\varepsilon}{2}
$$


Next choose $\delta>0$ such that if $d(p, y)<\delta$ then $|f(p)-f(y)|<\varepsilon / 2$. If $V$ denotes the open $\delta$ ball about $y$, there exists a neighbourhood $U$ of $x$ such that for each $z$ in $U$ $\Gamma_{\lambda}(z) \cap V \neq \varnothing$. Choose $n \geqq N$ so large that $x_{n} \in U$. Since $\Gamma_{\lambda}\left(x_{n}\right) \cap V \neq \varnothing$, there exists $p \in V$ such that $d\left(x_{n}, p\right) \leqq \lambda$. But this implies

$$
\begin{aligned}
f_{\lambda}^{+}\left(x_{n}\right) & =\sup _{w \in B_{\lambda}\left[x_{n}\right]} f(w)+\lambda \\
& \geqq f(p)+\lambda \geqq f(y)-\frac{\varepsilon}{2}+\lambda=f_{\lambda}^{+}(x)-\frac{\varepsilon}{2}
\end{aligned}
$$

This contradicts our choice of $N$ above.

Conversely, suppose $\Gamma_{\lambda}$ failed to be l.s.c. at some point $x$. There exists an open set $V$ meeting $\Gamma_{\lambda}(x)$ and a sequence $\left\{x_{n}\right\}$ convergent to $x$ such that for each $n \Gamma_{\lambda}\left(x_{n}\right) \cap V=\varnothing$. It is clear that for each $\beta<\lambda \Gamma_{\beta}(x) \cap V$ is empty, or else eventually $\left\{\Gamma_{\lambda}\left(x_{n}\right)\right\}$ would meet $V$. Thus

$$
\Gamma_{\lambda}(x) \cap V \subset\{y: d(x, y)=\lambda\}
$$

Next choose $z$ in $\Gamma_{\lambda}(x) \cap V$ and $\varepsilon>0$ such that $B_{\varepsilon}[z] \subset V$. Define $f \in C(X)$ by

$$
f(y)= \begin{cases}\varepsilon-d(z, y) & \text { if } y \in B_{\varepsilon}[z] \\ 0 & \text { otherwise }\end{cases}
$$

We claim that $f_{\lambda}^{+}$is discontinuous at $x$. First,

$$
\begin{aligned}
f_{\lambda}^{+}(x) & =\sup \left\{f(y)+\lambda: y \in B_{\lambda}[x]\right\} \\
& =\sup \left\{f(y)+\lambda: y \in B_{\lambda}[x] \cap B_{\varepsilon}[z]\right\} \\
& =\varepsilon+\lambda
\end{aligned}
$$

However, for each $n B_{\lambda}\left[x_{n}\right] \cap B_{\varepsilon}[z] \subset \Gamma_{\lambda}\left(x_{n}\right) \cap V=\varnothing$. Hence, for each $n f_{\lambda}^{+}\left(x_{n}\right)=0+\lambda=\lambda$ so that $\lim _{n \rightarrow \infty} f_{\lambda}^{+}\left(x_{n}\right) \neq f_{\lambda}^{+}(x)$. This concludes the proof.

To see that Lemma 4 is not content-free, we present a continuous function on a polygonally connected compact set in the plane that admits discontinuous upper parallel functions. Another somewhat misleading example appears in [3].

Example 3. In the plane with the usual metric let $X$ denote the union of the line segments joining $(0,0)$ to $(8,0)$ and $(0,0)$ to $(8,6)$. Define $f: X \rightarrow R$ by

$$
f\left(\alpha_{1}, \alpha_{2}\right)=\left\{\begin{array}{lll}
0 & \text { if } & \alpha_{2}=\frac{3}{4} \alpha_{1} \\
\alpha_{1} & \text { if } & \alpha_{2}=0
\end{array}\right.
$$

We claim that $f_{3}^{+}$is discontinuous at $(4,3)$. Since the closed disc with centre $(4,3)$ and 
radius 3 meets the line segment joining $(0,0)$ to $(8,0)$ in exactly one point, we have

$$
\begin{aligned}
f_{3}^{+}(4,3) & =\sup \left\{f\left(\alpha_{1}, \alpha_{2}\right)+3:\left(\alpha_{1}, \alpha_{2}\right) \in B_{3}[(4,3)]\right\} \\
& =\sup \left\{f\left(\alpha_{1}, \alpha_{2}\right)+3:\left(\alpha_{1}-4\right)^{2}+\left(\alpha_{2}-3\right)^{2} \leqq 9 \text { and }\left(\alpha_{1}, \alpha_{2}\right) \in X\right\} \\
& =f(4,0)+3=4+3=7
\end{aligned}
$$

However, if $1<\theta \leqq 2$ then the disc with centre $(4 \theta, 3 \theta)$ of radius 3 fails to meet the line segment. It follows that for all such $\theta f_{3}^{+}(4 \theta, 3 \theta)=3$, and the claim is established. Similar reasoning shows that if $0<\lambda<6$ then $f_{\lambda}^{+}$is discontinuous at $\left(\frac{4}{3} \lambda, \lambda\right)$.

Our next theorem is an immediate corollary of Theorem 2 and Lemma 4.

Theorem 3. Let $X$ be a metric space. Then each upper parallel function of each function in $C(X)$ is again in $C(X)$ if and only if each closed and bounded subset of $X$ is compact and each ball multifunction on $X$ is continuous.

To apply Theorem 3 in spaces in which closed and bounded subsets are compact, we need only check (by virtue of Lemma 2) that each ball multifunction is l.s.c. A Lechiki (private communication) has pointed out a simple sufficient (but not necessary) condition for the lower semicontinuity of each ball multifunction in an arbitrary metric space $X:$ if $x \in X$ and $\lambda>0$ and $\{y: d(x, y) \leqq \lambda\}=c l\{y: d(x, y)<\lambda\}$, then the ball multifunction $\Gamma_{\lambda}$ is 1.s.c. at $x$. To see this let $V \subset X$ be an open set that meets $\Gamma_{\lambda}(x)$. Then there exists a point $v$ in $V \cap\{y: d(x, y)<\lambda\}$. Choose $\varepsilon>0$ such that $\varepsilon<\lambda-d(x, v)$. Then if $d(z, x)<\varepsilon$, we have $d(z, v)<\lambda$, whence $\Gamma_{\lambda}(z) \cap V \neq \varnothing$. In particular, if $X$ is a convex subset of a normal linear space $L$, viewed as a metric subspace of $L$, then for each $\lambda>0$ the ball multifunction $\Gamma_{\lambda}$ is l.s.c. on $X$. Thus, if $X$ is a closed convex subset of $d$-dimensional Euclidean space $\mathbb{R}^{d}$, Theorem 3 ensures that each upper parallel function of each function in $C(X)$ is again in $C(X)$. Example 3 shows that an analogous statement cannot be made for compact starshaped subsets of $\mathbb{R}^{d}$.

A function $f: X \rightarrow(-\infty, \infty]$ defined on a convex subset $X$ of a normed linear space $L$ is called concave if for each $x, y$ in $X$ and each $\lambda \in[0,1]$ we have $f[\lambda x+(1-\lambda) y] \geqq \lambda f(x)$ $+(1-\lambda) f(y)$. Alternatively, $f$ is concave if its hypograph is a convex subset of $L \times R$. Since the parallel bodies of a convex set in a normed linear space are again convex sets [15], it is easy to see that if $f: X \rightarrow(-\infty, \infty]$ is a concave function, then so is each upper parallel function $f_{\lambda}^{+}$. The details are left to the reader.

Given a circle $C$ in the plane it is easy to see that each ball multifunction on $C$ is continuous: take $\delta=\varepsilon$. On the other hand, if $T$ is the boundary of a triangle, then for all $\lambda$ sufficiently small $\Gamma_{\lambda}$ fails to be globally continuous. To see this choose two sides of $T$ that enclose an acute angle and repeat the construction of Example 3. This author cannot characterise those plane convex curves for which each ball multifunction is continuous. Such a characterisation might not rest on an approximation by polygonal convex curves because the compact sets in the plane for which each ball multifunction is continuous do not form a closed set relative to the Hausdorff metric. In fact this collection is dense in the compact sets, for it includes all finite sets. 
Finally we present a characterisation of the closed subsets $X$ of $d$-dimensional Euclidean space $\mathbb{R}^{d}$ that have continuous ball multifunctions. In this theorem parallel bodies of sets are taken with respect to $\mathbb{R}^{d}$. We shall consider auxilliary multifunctions associated with $\Gamma_{\lambda}: X \rightarrow C L(X)$. For each $n \in Z^{+}$the multifunction $\Gamma_{\lambda}^{n}$ represents the $\lambda$-ball multifunction for the closed set $B_{1 / n}[X]$.

Theorem 4. Let $X$ be a closed subset of $\mathbb{R}^{d}$ and let $\lambda$ be positive. Then the ball multifunction $\Gamma_{\lambda}: X \rightarrow C L(X)$ is continuous if and only if the sequence $\left\{\Gamma_{\lambda}^{n} \mid X\right\}$ converges to $\Gamma_{\lambda}$ uniformly in the Hausdorff metric on compact subsets of $X$.

Proof. Fix $\lambda>0$. Suppose $\Gamma_{\lambda}$ fails to be 1.s.c. at some point $x$ in $X$. In terms of Berge's definition, there exists $\varepsilon>0$ and a sequence $\left\{x_{n}\right\}$ in $X$ such that for all $n$ $\left\|x_{n}-x\right\|<1 / n$ but $B_{2 \varepsilon}\left[\Gamma_{\lambda}\left(x_{n}\right)\right] \ngtr \Gamma_{\lambda}(x)$. For each $n$ choose $y_{n}$ in $\Gamma_{\lambda}(x)-B_{2 \varepsilon}\left[\Gamma_{\lambda}\left(x_{n}\right)\right]$; clearly, $B_{\varepsilon}\left[y_{n}\right] \cap B_{\varepsilon}\left[\Gamma_{\lambda}\left(x_{n}\right)\right]=\varnothing$. Next choose $N$ so large that $1 / N<\varepsilon$. By the choice of $N$, for each $n \geqq N$ the point $z_{n}=y_{n}+\left(x_{n}-x\right)$ lies in $B_{\varepsilon}\left[y_{n}\right]$ and

$$
\left\|z_{n}-x_{n}\right\|=\left\|y_{n}-x\right\| \leqq \lambda
$$

Since $z_{n} \in B_{1 / n}\left[\Gamma_{\lambda}(x)\right] \subset B_{1 / n}[X]$ it follows that $z_{n} \in \Gamma_{\lambda}^{n}\left(x_{n}\right)$. Since $z_{n} \in B_{\varepsilon}\left[y_{n}\right]$ we also know that for each $n \geqq N \Gamma_{\lambda}^{n}\left(x_{n}\right) \not B_{\varepsilon}\left[\Gamma_{\lambda}\left(x_{n}\right)\right]$. Thus the Hausdorff distance of $\Gamma_{\lambda}^{n}\left(x_{n}\right)$ from $\Gamma_{\lambda}\left(x_{n}\right)$ exceeds $\varepsilon$ for all $n \geqq N$ so that $\left\{\Gamma_{\lambda}^{n} \mid X\right\}$ fails to converge uniformly to $\Gamma_{\lambda}$ on $\{x\} \cup\left\{x_{n}: n \in Z^{+}\right\}$, a compact subset of $X$.

The converse, i.e., that continuity of $\Gamma_{\lambda}$ implies uniform convergence on compact subsets of $X$, follows immediately from Dini's theorem for multifunctions [2] because (i) for each $n B_{1 / n}[X]$ is a closed subset of $\mathbb{R}^{d}$ so that $\Gamma_{\lambda}^{n}$ is u.s.c., and (ii) for each $x$ in $X$ and for all $n \Gamma_{\lambda}^{n}(x) \supset \Gamma_{\lambda}^{n+1}(x)$ and $\bigcap_{n=1}^{\infty} \Gamma_{\lambda}^{n}(x)=\Gamma_{\lambda}(x)$

We close by mentioning that there is some literature on the structure of the boundary of parallel bodies of more general sets. We refer the interested reader to Gindifer [10], Brown [6], Gariepy and Pepe [9\}, Beer [1], and Ferry [8].

\section{REFERENCES}

1. G. Beer, Starshaped sets and the Hausdorff metric, Pacific J. Math. 61 (1975), 21-27.

2. G. BEER, The approximation of upper semicontinuous multifunctions by step multifunctions, Pacific J. Math. 87 (1980), 11-19.

3. G. BEER, Upper semicontinuous functions and the Stone approximation theorem, $J$. Approximation Theory 34 (1982), 1-11.

4. C. Berge, Topological spaces (Oliver and Boyd, Edinburgh, 1963).

5. C. Borges, A study of multivalued functions, Pacific J. Math 23 (1967), 451-461.

6. M. Brown, Sets of constant distance from a planar set, Michigan Math. J. 19 (1972), 321-323.

7. S. Dolecki and S. Rolewicz, Metric characterizations of upper semicontinuity, J. Math. Anal. Appl. 69 (1979), 146-152.

8. S. FerRY, When $\varepsilon$-boundaries are manifolds, Fund. Math. 90 (1976), 199-210. 
9. R. Gariepy and W. Pepe, On the level sets of a distance function in a Minkowski space, Proc. Amer. Math. Soc. 31 (1972), 255-259.

10. M. GINDIFER, On generalized spheres, Fund. Math. 38 (1951), 167-178.

11. G. Goudin,, On set-values mappings, Rev. Roum. Math. Pures Appl. 22 (1) (1977), 53-67.

12. N. Hindman, Basically bounded sets and a generalised Heine-Borel theorem, Amer. Math. Monthly 80 (1973), 549-552.

13. S. Hu, Introduction to general topology (Holden Day, San Francisco, 1966).

14. S. NAdLer, Hyperspaces of sets (Marcel Dekker, New York, 1978).

15. F. Valentine, Convex sets, (McGraw-Hill, New York, 1964).

California State University, Los Angeles

Los Angeles, California 90032 